\title{
TWO ESTIMATES CONCERNING CLASSICAL DIOPHANTINE APPROXIMATION CONSTANTS
}

\author{
JOHANNES SCHLEISCHITZ
}

\begin{abstract}
In this paper we aim to prove two inequalities involving the classical approximation constants $w_{n}^{\prime}(\zeta), \widehat{w}_{n}^{\prime}(\zeta)$ that stem from the simultaneous approximation problem $\left|\zeta^{j} x-y_{j}\right|, 1 \leq j \leq n$, on the one side and the constants $w_{n}^{*}(\zeta), \widehat{w}_{n}^{*}(\zeta)$ connected to approximation with algebraic numbers of degree $\leq n$ on the other side. We concretely prove $w_{n}^{*}(\zeta) \widehat{w}_{n}^{\prime}(\zeta) \geq 1$ and $\widehat{w}_{n}^{*}(\zeta) w_{n}^{\prime}(\zeta) \geq 1$. The first result is due to W. Schmidt, however our method of proving it allows to derive the other inequality as a dual result. Finally we will discuss estimates of $w_{n}^{*}(\zeta), \widehat{w}_{n}^{*}(\zeta)$ uniformly in $\zeta$ depending only on $n$ as an application.
\end{abstract}

AMS 2010 Mathematics Subject Classification: 11J13, $11 \mathrm{H} 06$

Supported by FWF grant P22794-N13

\section{INTRODUCTION}

1.1. Approximation constants $w_{n}^{\prime}(\zeta), \widehat{w}_{n}^{\prime}(\zeta)$ and $w_{n}^{*}(\zeta), \widehat{w}_{n}^{*}(\zeta)$. For a fixed positive integer $n$ and a vector $\zeta=\left(\zeta_{1}, \zeta_{2}, \ldots, \zeta_{n}\right) \in \mathbb{R}^{n}$ define the approximation constants $w_{n, j}^{\prime}(\zeta), 1 \leq$ $j \leq n+1$, as the supremum of all real numbers $\nu$, such that the system

$$
|x| \leq X, \quad\left|\zeta_{i} x-y_{i}\right| \leq X^{-\nu}, \quad 1 \leq i \leq n,
$$

has $j$ linearly independent solution $\left(x, y_{1}, \ldots, y_{n}\right)$ for certain arbitrary large values of $X$. Similarly, define $\widehat{w}_{n, j}^{\prime}(\zeta), 1 \leq j \leq n+1$ as the supremum of all $\nu$, such that system (1.1) has $j$ linearly independent solutions for all sufficiently large $X$. Clearly $\widehat{w}_{n, j}^{\prime}(\zeta) \leq w_{n, j}^{\prime}(\zeta)$ for all $1 \leq j \leq n+1$. In the special case $\zeta=\left(\zeta, \zeta^{2}, \ldots, \zeta^{n}\right)$ for some real number $\zeta$, which will be in the focus of our study, let $w_{n, j}^{\prime}(\zeta):=w_{n, j}^{\prime}(\boldsymbol{\zeta})$ and similarly $\widehat{w}_{n, j}^{\prime}(\zeta):=\widehat{w}_{n, j}(\boldsymbol{\zeta})$. For convenient writing we further put $w_{n}^{\prime}(\boldsymbol{\zeta}):=w_{n, 1}^{\prime}(\boldsymbol{\zeta}), \widehat{w}_{n}^{\prime}(\boldsymbol{\zeta}):=\widehat{w}_{n, 1}^{\prime}(\boldsymbol{\zeta})$. In particular $w_{n}^{\prime}(\zeta)$ (resp. $\left.\widehat{w}_{n}^{\prime}(\zeta)\right)$ is the supremum of all $\nu$ such that (1.1) with $\zeta_{i}=\zeta^{i}$ has infinitely many solutions respectively a solution for all sufficiently large $X$.

The approximation constants $w_{n}^{*}(\zeta), \widehat{w}_{n}^{*}(\zeta)$ quantify how good a real number can be approximated by algebraic numbers of degree at most $n$. For a polynomial $P \in \mathbb{Z}[T]$ define $H(P)$ as the largest absolute value of its coefficients and for an algebraic number $\alpha$ define $H(\alpha):=H(P)$ for the minimal polynomial $P$ of $\alpha$ with relatively prime integral coefficients. The constants $w_{n}^{*}(\zeta)$ (resp. $\left.\widehat{w}_{n}^{*}(\zeta)\right)$ are given by the supremum of all real $\nu$ such that

$$
|\zeta-\alpha| \leq H(\alpha)^{-\nu-1}
$$

has infintely many solutions, respectively a solution for arbitrarily large values of $H(\alpha)$, with $\alpha$ algebraic of degree $\leq n$. Clearly $w_{n}^{*}(\zeta), \widehat{w}_{n}^{*}(\zeta)$ are monotonically increasing as $n$ increases. For $w_{n}^{*}(\zeta)$ it is known that we have

$$
\frac{n+1}{2} \leq w_{n}^{*}(\zeta)
$$

for all $\zeta \in \mathbb{R}$ not algebraic of degree $\leq n$ as a consequence of (1.9) and (2.20) we will establish later.

It is however conjectured that even the stronger lower bound $w_{n}^{*}(\zeta) \geq n$ holds for any $\zeta$ not 
algebraic of degree $\leq n$. It is well known that $n$ is the optimal possible uniform (in $\zeta$ ) lower bound in (1.3), as for a generic $\zeta$ we have $w_{n}^{*}(\zeta)=\widehat{w}_{n}^{*}(\zeta)=n$, see Theorem 2.3 in [1] for example.

1.2. Related approximation problems and constants. We will only treat the case $\boldsymbol{\zeta}=\left(\zeta, \zeta^{2}, \ldots, \zeta^{n}\right)$ in the sequel which is sufficient for our concern, although most of the following results in the introduction hold for any vector $\zeta \in \mathbb{R}^{n}$.

In order to study the values $w_{n}^{\prime}(\zeta), \widehat{w}_{n}^{\prime}(\zeta)$ we first introduce a closely connected Diophantine approximation problem. Consider the system

$$
\begin{aligned}
|x| & \leq Q^{1+\theta} \\
\left|\zeta x-y_{1}\right| & \leq Q^{-\frac{1}{n}+\theta} \\
\left|\zeta^{2} x-y_{2}\right| & \leq Q^{-\frac{1}{n}+\theta} \\
\vdots & \vdots \\
\left|\zeta^{n} x-y_{n}\right| & \leq Q^{-\frac{1}{n}+\theta}
\end{aligned}
$$

parametrised by $Q>1$. Let $\psi_{n, j}(Q)$ be the infimum of all $\theta$ such that (1.4) has $j$ linearly independent solutions. The functions $\psi_{n, j}(Q)$ can alternatively be interpreted via successive minima. Consider the lattice $\Lambda=\left\{\left(x, \zeta x-y_{1}, \ldots, \zeta^{n} x-y_{n}\right): x, y_{1}, \ldots y_{n} \in \mathbb{Z}\right\}$ and the convex body (in fact the parallelepiped) $K(Q)$ defined as the set of points $\left(z_{1}, z_{2}, \ldots, z_{n+1}\right) \in$ $\mathbb{R}^{n+1}$ with

$$
\begin{aligned}
& \left|z_{1}\right| \leq Q \\
& \left|z_{i}\right| \leq Q^{-\frac{1}{n}}, \quad 2 \leq i \leq n+1 .
\end{aligned}
$$

Now define $\lambda_{n, j}(Q)$ to be the $j$-th successive minimum of $K(Q)$ with respect to $\Lambda$, which by definiton is the smallest value $\lambda$ such that $\lambda \cdot K(Q)$ contains (at least) $j$ linearly independent lattice points. Then we have

$$
Q^{\psi_{n, j}(Q)}=\lambda_{n, j}(Q)
$$

Put $\underline{\psi}_{n, j}:=\liminf \lim _{Q \rightarrow \infty} \psi_{n, j}(Q), \bar{\psi}_{n, j}:=\limsup _{Q \rightarrow \infty} \psi_{n, j}(Q)$. We have $-1 \leq \psi_{n, j}(Q) \leq \frac{1}{n}$ for all $Q>1$ and $1 \leq j \leq n+1$ (which is implicitely derived rigurously from section 4 in 6 . considering the functions $L_{j}$ arising from $\psi_{n, j}$ ) and consequently

$$
\begin{aligned}
-1 & \leq \underline{\psi}_{n, 1} \leq \underline{\psi}_{n, 2} \leq \cdots \leq \underline{\psi}_{n, n+1} \leq \frac{1}{n} \\
-1 & \leq \bar{\psi}_{n, 1} \leq \bar{\psi}_{n, 2} \leq \cdots \leq \bar{\psi}_{n, n+1} \leq \frac{1}{n}
\end{aligned}
$$

A crucial observation for the study of the functions $\psi_{n, j}(Q)$ (a special case of Theorem 1.1 in [6]) is that for $\zeta$ not algebraic of degree $\leq n$ and every $1 \leq j \leq n$ there are infinitely many $Q$ with $\psi_{n, j}(Q)=\psi_{n, j+1}(Q)$. Thus in particular

$$
\underline{\psi}_{n, j+1} \leq \bar{\psi}_{n, j}, \quad 1 \leq j \leq n .
$$

An easy generalisation of Theorem 1.4 in [6] states that we have

$$
\left(1+\omega_{n, j}^{\prime}(\zeta)\right)\left(1+\underline{\psi}_{n, j}\right)=\left(1+\widehat{\omega}_{n, j}^{\prime}(\zeta)\right)\left(1+\bar{\psi}_{n, j}\right)=\frac{n+1}{n}, \quad 1 \leq j \leq n+1,
$$

which enables us to easily compute the value $\underline{\psi}_{n, j}$ from $w_{n, j}^{\prime}(\zeta)$ such as $\bar{\psi}_{j}$ from $\widehat{w}_{n, j}^{\prime}(\zeta)$ and vice versa.

The approximation constants $w_{n}^{*}(\zeta), \widehat{w}_{n}^{*}(\zeta)$ are in close connection to the dual problem of approximation of a linear form, which in our case is just $x+\zeta y_{1}+\ldots+\zeta^{n} y_{n}$.

First, define the approximation constants $w_{n, j}(\zeta)$ (resp. $\left.\widehat{w}_{n, j}(\zeta)\right)$ as the supremum of all $\nu \in \mathbb{R}$, such that the system

$$
\left|y_{i}\right| \leq X, \quad\left|x+\zeta y_{1}+\ldots+\zeta^{n} y_{n}\right| \leq X^{-\nu}
$$


has $j$ linearly independent solutions $\left(x, y_{1}, \ldots, y_{n}\right)$ for certain arbitrary large values of $X$ (respectively all sufficiently large $X$ ) and put $w_{n}(\zeta):=w_{n, 1}(\zeta), \widehat{w}_{n}(\zeta):=\widehat{w}_{n, 1}(\zeta)$. In virtue of Dirichlet's Theorem we have

$$
w_{n}(\zeta) \geq \widehat{w}_{n}(\zeta) \geq n
$$

Given the dual lattice $\Lambda^{*}$ and the convex bodies $K^{*}(Q)$ dual to $K(Q)$, namely

$$
\begin{aligned}
(1.10) \quad \Lambda^{*} & :=\left\{\left(x+\zeta_{1} y_{1}+\zeta_{2} y_{2}+\cdots+\zeta_{n} y_{n}, y_{1}, y_{2}, \ldots, y_{n}\right): x, y_{1}, \ldots, y_{n} \in \mathbb{Z}\right\} \\
(1.11) K^{*}(Q) & :=\left\{\boldsymbol{x}=\left(x, y_{1}, \ldots, y_{n}\right): \quad<\boldsymbol{x}, \boldsymbol{z}>\leq 1 \quad \forall \boldsymbol{z} \in K(Q)\right\}
\end{aligned}
$$

we can define the functions $\lambda_{n, j}^{*}(Q)$ as the successive minima of $K^{*}(Q)$ with respect to $\Lambda^{*}$. Put

$$
Q^{\psi_{n, j}^{*}(Q)}=\lambda_{n, j}^{*}(Q) .
$$

Furthermore we denote by $\eta_{n, j}^{*}(Q)$ the successive minima of $K(Q)$ (instead of $K^{*}(Q)$ ) with respect to $\Lambda^{*}$, which correspond to the successive minima of the convex body $K^{+}(Q)$ with respect to the lattice $\Lambda^{+}$given by

$$
(1.13) K^{+}(Q):=\quad\left\{\boldsymbol{x} \in \mathbb{R}^{n+1}:\left|y_{t}\right| \leq Q^{\frac{1}{n}} \quad 1 \leq t \leq n,\left|x+\zeta y_{1}+\cdots+\zeta^{n} y_{n}\right| \leq Q^{-1}\right\}
$$

and define functions $\nu_{n, j}^{*}(Q)$ by $Q^{\nu_{n, j}^{*}(Q)}=\eta_{n, j}^{*}(Q)$. If we put $\underline{\psi}_{n, j}^{*}:=\liminf \operatorname{in}_{Q \rightarrow \infty} \psi_{n, j}^{*}(Q), \bar{\psi}_{n, j}^{*}:=$ $\lim \sup _{Q \rightarrow \infty} \psi_{n, j}^{*}(Q)$ and similarly define $\underline{\nu}_{n, j}^{*}, \bar{\nu}_{n, j}^{*}$ the obvious inequalities $K^{*}(Q) \subset K(Q) \subset$ $(n+1) K^{*}(Q)$ yield

$$
\lim _{Q \rightarrow \infty} \psi_{n, j}^{*}(Q)-\nu_{n, j}^{*}(Q)=0
$$

and thus

$$
\underline{\psi}_{n, j}^{*}=\underline{\nu}_{n, j}^{*}, \quad \bar{\psi}_{n, j}^{*}=\bar{\nu}_{n, j}^{*} .
$$

Combined with Mahler's inequality $\lambda_{n, j} \lambda_{n, n+2-j}^{*} \asymp 1$ for $1 \leq j \leq n+1$ we have

$$
\underline{\psi}_{n, j}=-\bar{\psi}_{n, n+2-j}^{*}=-\bar{\nu}_{n, n+2-j}^{*}, \quad \bar{\psi}_{n, j}=-\underline{\psi}_{n, n+2-j}^{*}=-\underline{\nu}_{n, n+2-j}^{*} .
$$

It will be more convenient to work with the functions $\nu_{n, j}^{*}$ in the sequel. Again by generalizing Theorem 1.4 in [6] we have

$$
\left(w_{n, j}(\zeta)+1\right)\left(\frac{1}{n}+\underline{\psi}_{n, j}^{*}\right)=\left(\widehat{w}_{n, j}(\zeta)+1\right)\left(\frac{1}{n}+\bar{\psi}_{n, j}^{*}\right)=\frac{n+1}{n}
$$

for $1 \leq j \leq n+1$, and obviously by (1.15) we can write $\underline{\nu}_{j}^{*}$ resp. $\bar{\nu}_{j}^{*}$ instead of $\underline{\psi}_{j}^{*}$ resp. $\bar{\psi}_{j}^{*}$. A main ingredient in the proof of the Theorem 1.2 will be Minkowski's Theorem, which asserts that for a convex body $K \in \mathbb{R}^{n+1}$, a lattice $\Lambda$ and corresponding successive minima $\lambda_{n, j}$ we have

$$
\frac{2^{n+1}}{(n+1) !} \frac{\operatorname{det}(\Lambda)}{\operatorname{vol}(\mathrm{K})} \leq \lambda_{n, 1} \lambda_{n, 2} \cdots \lambda_{n, n+1} \leq 2^{n+1} \frac{\operatorname{det}(\Lambda)}{\operatorname{vol}(\mathrm{K})} .
$$

For a proof see Theorem 1 page 60 and Theorem 2 page 62 in [3].

In our special case the height of the parallelepipeds $K^{+}(Q)$ in direction of the $x$-axis in every point $\left(x, y_{1}, \ldots, y_{n}\right)$ with $\left(y_{1}, y_{2}, \ldots, y_{n}\right) \in\left[-Q^{\frac{1}{n}}, Q^{\frac{1}{n}}\right]^{n}$ is $2 Q^{-1}$, so we have $\operatorname{vol}\left(\mathrm{K}^{+}(\mathrm{Q})\right)=$ $\left(2 \mathrm{Q}^{\frac{1}{n}}\right)^{\mathrm{n}} \cdot 2 \mathrm{Q}^{-1}=2^{\mathrm{n}+1}$ for all $Q>1$, and furthermore $\operatorname{det}\left(\Lambda^{+}\right)=\operatorname{det}\left(\mathbb{Z}^{n+1}\right)=1$. Since $\eta_{n, j}^{*}$ are the successive minima of $K^{+}(Q)$ with respect to $\Lambda^{+}$, (1.18) leads to

$$
\frac{1}{(n+1) !} \leq \eta_{n, 1}^{*}(Q) \eta_{n, 2}^{*}(Q) \cdots \eta_{n, n+1}^{*}(Q) \leq 1
$$


Hence by taking logarithms, there is a constant $C$ depending only on $n$ such that

$$
\left|\sum_{i=1}^{n+1} \nu_{n, i}^{*}(Q)\right| \leq \frac{C(n)}{\log (Q)} .
$$

The constants $w_{n}^{*}(\zeta), \widehat{w}_{n}^{*}(\zeta)$ are closely linked to the constants $w_{n, j}(\zeta), \widehat{w}_{n, j}(\zeta)$, as already indicated above. For instance we have $\frac{w_{n}(\zeta)+1}{2} \leq w_{n}^{*}(\zeta) \leq w_{n}(\zeta)$, which by (1.9) implies the bound (1.3) for all $\zeta$ mentioned in subsection 1.1. See also remark 3 to Corollary 2.2 in section 2.2. In fact, we will prove

Theorem 1.1. For any integer $n \geq 1$ and any $\zeta \in \mathbb{R}$ not algebraic of degree $\leq n$ we have

$$
\begin{aligned}
& w_{n}^{*}(\zeta) \geq w_{n, n+1}(\zeta) \\
& \widehat{w}_{n}^{*}(\zeta) \geq \widehat{w}_{n, n+1}(\zeta) .
\end{aligned}
$$

Note that by (1.8), (1.16), (1.17) one can directly compute $w_{n, n+2-j}^{\prime}(\zeta)$ from $\widehat{w}_{n, j}(\zeta)$ as well as $\widehat{w}_{n, n+2-j}^{\prime}(\zeta)$ from $w_{n, j}(\zeta)$ for $1 \leq j \leq n+1$, which leads to

$$
w_{n, n+2-j}^{\prime}(\zeta)=\frac{1}{\widehat{w}_{n, j}(\zeta)}, \quad \widehat{w}_{n, n+2-j}^{\prime}(\zeta)=\frac{1}{w_{n, j}(\zeta)}
$$

Combining (1.22) with Theorem 1.1 we immediately obtain

Theorem 1.2. For any integer $n \geq 1$ and any $\zeta \in \mathbb{R}$ not algebraic of degree $\leq n$ we have

$$
\begin{aligned}
& w_{n}^{*}(\zeta) \geq \frac{1}{\widehat{w}_{n}^{\prime}(\zeta)} \\
& \widehat{w}_{n}^{*}(\zeta) \geq \frac{1}{w_{n}^{\prime}(\zeta)}
\end{aligned}
$$

It remains to prove Theorem 1.1. A basic observation linking $w_{n}^{*}(\zeta)$ with the constants $w_{n, j}(\zeta)$ is the fact that any non-zero polynomial $P \in \mathbb{Z}[T]$ with $P^{\prime}(\zeta) \neq 0$ has a root $\alpha$ satisfying

$$
|\zeta-\alpha| \leq n\left|\frac{P(\zeta)}{P^{\prime}(\zeta)}\right|
$$

since for $P(\zeta)=\prod_{1 \leq i \leq n}\left(\zeta-\alpha_{i}\right)$ with $\alpha$ the closest zero to $\zeta$ (i.e. minimizing $|\zeta-\alpha|$ ) we have $\left|\frac{P^{\prime}(\zeta)}{P(\zeta)}\right|=\left|\sum_{1 \leq i \leq n} \frac{1}{\zeta-\alpha_{i}}\right| \leq n \max _{1 \leq i \leq n} \frac{1}{\left|\zeta-\alpha_{i}\right|}=n \frac{1}{|\zeta-\alpha|}$. So in order to get a sequence of good apprimation values $\alpha$ for a fixed $\zeta$, we only need to find a sequence of polynomials with small values $\left|\frac{P(\zeta)}{P^{\prime}(\zeta)}\right|$. Note that the logarithm of $P(\zeta)$ to the basis $H(P)$ (see section $1.1)$ is directly connected to $w_{n}(\zeta), \widehat{w}_{n}(\zeta)$. Putting

$$
w_{n}^{*}(\zeta, H):=\min _{P: H(P) \leq H}-\frac{\log \left|\frac{P(\zeta)}{P^{\prime}(\zeta)}\right|}{\log H}-1
$$

where for all fixed $H, P$ runs through all polynomials $P$ of height $H(P) \leq H$, by virtue of (1.23) one easily deduces

$$
\begin{aligned}
& w_{n}^{*}(\zeta) \geq \limsup _{H \rightarrow \infty} w_{n}^{*}(\zeta, H) \\
& \widehat{w}_{n}^{*}(\zeta) \geq \liminf _{H \rightarrow \infty} w_{n}^{*}(\zeta, H) .
\end{aligned}
$$




\section{Proof of TheOREM 1.1}

2.1. Strategy of the proof. In fixed dimension $n$ we consider two successive minima problems. On the one hand the successive minima $\eta_{n, j}^{*}(Q)$ of the bodies $K^{+}(Q)$ with respect to the lattice $\Lambda^{+}$defined in (1.12), (1.13) and the resulting functions $\nu_{n, j}^{*}(Q)$ arising from $\eta_{n, j}^{*}(Q)$ from section 1.2. On the other hand, we compress the bodies $K^{+}(Q)$ in direction orthogonal to the hyperplane $P^{\prime}(\zeta)=0$ by a fixed factor and consider their successive minima with respect to $\Lambda^{+}$. By applying Minkowski's Theorem (1.18) to both systems we will infer that at least one successive minimum of these two systems must differ from the corresponding successive minimum of the other system. This will imply the existence of lattice points (in fact points corresponding to a successive minimum!) with relatively "large" values $\left|P^{\prime}(\zeta)\right| \approx H(P)$, which is helpful for lower bounds for $w_{n}^{*}(\zeta), \widehat{w}_{n}^{*}(\zeta)$ in view of (1.23) (or (1.24) ). Assuming this point corresponds to the last (i.e. $(n+1)$-st) successive minimum gives a lower estimate for $w_{n}^{*}(\zeta), \widehat{w}_{n}^{*}(\zeta)$ and together with the very intuitive geometric Lemma 2.1 (although slightly techincal to prove) concerning the volume of the compressed bodies, (1.17) and (1.25) resp. (1.26) leads to the estimates (1.20) resp. (1.21) in Theorem 1.1.

2.2. Exact proof of Theorem 1.1. We will assume $\zeta$ fixed and identify a point $P=$ $\left(x, y_{1}, \ldots, y_{n}\right) \in \mathbb{Z}^{n+1}$ with the polynomial $P(\zeta)=x+\zeta y+\ldots+\zeta^{n} y_{n}$. For technical reasons we will call the successive minima problem concerning $K^{+}(Q)$ and $\Lambda^{+}$system $A$ throughout section 2. We denote it with superscript $A$ and for simplicity we write $\eta_{n, j}^{A}(Q):=$ $\eta_{n, j}^{*}(Q), \nu_{n, j}^{A}(Q):=\nu_{n, j}^{*}(Q)$ with $\eta_{n, j}^{*}(Q), \nu_{n, j}^{*}(Q)$ as defined in section 1.2.

Furthermore, define system $B$ as the successive minimum problem concerning $\Lambda^{+}$and the convex body

with

$$
\mathscr{K}(Q):=K^{+}(Q) \cap c(Q) \cdot W(Q)
$$

$$
W(Q):=\left\{\left(x, y_{1}, \ldots, y_{n}\right) \in \mathbb{R}^{n+1}:\left|y_{1}+2 \zeta y_{2}+\cdots+n \zeta^{n-1} y_{n}\right| \leq Q^{\frac{1}{n}}\right\}
$$

and positive real numbers $c(Q)$ to be chosen later. Observe that $W(Q)$ is just the set of points $P \in \mathbb{R}^{n+1}$ with $\left|P^{\prime}(\zeta)\right| \leq Q^{\frac{1}{n}}$, so the convex bodies $K^{+}(Q)$ are "somehow compressed" by some factor $c(Q)$ in the direction orthogonal to the hyperplane $P^{\prime}(\zeta)=0$ (although this is not quite true as the boundary changes shape). Successive minima functions $\eta_{n, j}^{B}(Q), \nu_{n, j}^{B}(Q)$ arise from system B similarly as in system A. Note that by construction we have $\mathscr{K}(Q) \subset K^{+}(Q)$ and therefore $\eta_{n, j}^{B}(Q) \leq \eta_{n, j}^{A}(Q)$ and $\nu_{n, j}^{B}(Q) \leq \nu_{n, j}^{A}(Q)$ for every $1 \leq j \leq n+1$ and $Q>1$.

We now choose the constants $c(Q)$ in (2.1) such that

$$
\operatorname{vol}(\mathscr{K}(\mathrm{Q}))=\frac{1}{2(\mathrm{n}+1) !} \operatorname{vol}\left(\mathrm{K}^{+}(\mathrm{Q})\right)<\frac{1}{(\mathrm{n}+1) !} \operatorname{vol}\left(\mathrm{K}^{+}(\mathrm{Q})\right)=\frac{2^{\mathrm{n}+1}}{(\mathrm{n}+1) !} .
$$

Clearly, this is possible as the volume of $\mathscr{K}(Q)$ with arbitrary $c(Q)$ in (2.1) depends continuously on $c(Q)$ and for sufficiently large $c(Q) \geq c_{0}(Q)$ we have $K^{+}(Q)=\mathscr{K}(Q)$, so in particular $\operatorname{vol}\left(\mathrm{K}^{+}(\mathrm{Q})\right)=\operatorname{vol}(\mathscr{K}(\mathrm{Q}))$, as well as $\operatorname{vol}(\mathscr{K}(\mathrm{Q}))=0$ for $c(Q)=0$. By the intermediate value theorem and as the volume increases strictly as $c(Q)$ increases (as long as $\left.\mathscr{K}(Q) \subsetneq K^{+}(Q)\right)$, there is a unique $c(Q)$ with (2.2) for every $Q>1$.

By (1.18) we infer that for any $Q>1$, for at least one $k=k(Q) \in\{1,2, \ldots, n+1\}$ we have strict inequality $\eta_{n, k}^{B}(Q)<\eta_{n, k}^{A}(Q)$. By the definition of successive minima and the choice of our convex bodies $\mathscr{K}(Q)$ and $K^{+}(Q)$ this gives the existence of vectors $\boldsymbol{d}(Q) \in \mathbb{Z}^{n+1}$ with

$$
\boldsymbol{d}(Q) \in\left(Q^{\nu_{n, k}^{A}(Q)} K^{+}(Q)\right) \backslash\left(Q^{\nu_{n, k}^{B}(Q)} \mathscr{K}(Q)\right)
$$

We will again identify any such $\boldsymbol{d}(Q)=\left(x, y_{1}, \ldots, y_{n}\right)$ with the corresponding polynomial $P(\zeta)=x+\zeta y_{1}+\ldots+\zeta^{n} y_{n}$. We consider these polynomials $P(\zeta)$ as $Q$ increases and will drop the dependence of $P$ from $Q$ in the notation as no misunderstandings can occur. Since $\mathscr{K}(Q)$ only differs from $K^{+}(Q)$ in direction orthogonal to $P^{\prime}(\zeta)=0$ we have

$$
\left|P^{\prime}(\zeta)\right|>Q^{\nu_{n, k}^{A}(Q)} \cdot c(Q) Q^{\frac{1}{n}}=c(Q) Q^{\nu_{n, k}^{A}(Q)+\frac{1}{n}}
$$


On the other hand, as $\boldsymbol{d}(Q) \in Q^{\nu_{n, j}^{A}(Q)} K^{+}(Q)$ we have

$$
\begin{aligned}
|P(\zeta)| & \leq Q^{-1+\nu_{n, k}^{A}(Q)}, \\
H(P) & \ll Q^{\frac{1}{n}+\nu_{n, k}^{A}(Q)} .
\end{aligned}
$$

with constants in $\ll$ depending only on $n, \zeta$. More precisely, as by defintion we have $\left|y_{t}\right| \leq$ $Q^{\frac{1}{n}+\nu_{n, k}^{A}(Q)}$ for $1 \leq t \leq k$ and clearly $|P(\zeta)| \leq 1$ for $Q$ sufficiently large, we have the estimates

$$
|x| \leq|P(\zeta)|+\left(1+|\zeta|+\cdots+|\zeta|^{n}\right) \max _{1 \leq t \leq n}\left|y_{t}\right| \leq 1+\left(1+|\zeta|+\cdots+|\zeta|^{n}\right) Q^{\frac{1}{n}+\nu_{n, k}^{A}(Q)} .
$$

So we infer $H(P) \leq 1+\left(1+|\zeta|+\cdots+|\zeta|^{n}\right) Q^{\frac{1}{n}+\nu_{n, k}^{A}(Q)}$ and thus (2.5).

Note that (2.3), (2.4), (2.5) hold for any large $Q$ and $\nu_{n, k}^{A}(Q) \leq \nu_{n, n+1}^{A}(Q)$ as well as $\underline{\nu}_{n, n+1}^{A}-$ $\epsilon \leq \nu_{n, n+1}(Q) \leq \bar{\nu}_{n, n+1}+\epsilon$ for all $\epsilon>0$ and $Q \geq Q(\epsilon)$. So on the one hand we can choose a sequence of values $\left(Q_{s}\right)_{s \geq 1} \rightarrow \infty$ such that the corresponding polynomials for any $\epsilon>0$ and sufficiently large $Q \geq \bar{Q}_{0}(\epsilon)$ satisfy

$$
\begin{aligned}
|P(\zeta)| & \leq Q^{-1+\underline{\nu}_{n, n+1}^{A}+\epsilon} \\
\left|P^{\prime}(\zeta)\right| & \geq c(Q) Q^{\frac{1}{n}+\underline{\underline{L}}_{n, n+1}^{A}} \\
H(P) & \ll Q^{\frac{1}{n}+\underline{\perp}_{n, n+1}^{A}}
\end{aligned}
$$

with constants depending only on $n, \zeta$ in $\ll$.

On the other hand, for any sufficiently large $Q \geq Q_{0}(\epsilon)$ we clearly have

$$
\begin{aligned}
|P(\zeta)| & \leq Q^{-1+\bar{\nu}_{n, n+1}^{A}+\epsilon} \\
\left|P^{\prime}(\zeta)\right| & \geq c(Q) Q^{\frac{1}{n}+\bar{\nu}_{n, n+1}^{A}} \\
H(P) & \ll Q^{\frac{1}{n}+\bar{\nu}_{n, n+1}^{A}} .
\end{aligned}
$$

Assume in our present situation, i.e. $c(Q)$ defined by (2.1), (2.2), we already knew

$$
\liminf _{Q \rightarrow \infty} \log _{Q} c(Q) \geq 0,
$$

which will be shown in Corollary 2.2 from Lemma 2.1. Then a choice of polynomials leading to (2.7),(2.8),(2.9) gives in combination with (1.24),(1.25) and $\epsilon \rightarrow 0$

$$
w_{n}^{*}(\zeta)+1 \geq-\frac{\log \left(\frac{P(\zeta)}{P^{\prime}(\zeta)}\right)}{\log (H(P))} \geq \frac{n+1}{n} \frac{1}{\frac{1}{n}+\underline{\nu}_{n, n+1}^{A}}=w_{n, n+1}(\zeta)+1,
$$

the equality on the right being just a variaton of (1.17). Similarly (2.10), (2.11),(2.12) gives in combination with (1.24), (1.26), (1.17) and $\epsilon \rightarrow 0$

$$
\widehat{w}_{n}^{*}(\zeta)+1 \geq-\frac{\log \left(\frac{P(\zeta)}{P^{\prime}(\zeta)}\right)}{\log (H(P))} \geq \frac{n+1}{n} \frac{1}{\frac{1}{n}+\bar{\nu}_{n, n+1}^{A}}=\widehat{w}_{n, n+1}(\zeta)+1 .
$$

Subtracting one from both sides of (2.14), (2.15) establishes the assertions of Theorem 1.1. It remains to prove (2.13). In fact, we prove that $c(Q)$ is even bounded below uniformly in the parameter $Q$.

Lemma 2.1. [Geometric lemma]

Given $n \geq 2, \zeta \in \mathbb{R}$ as well as positive real parameters $R$ and $Q>1$, consider the sets

$$
\begin{aligned}
& \chi_{A}(Q):=\left\{\left(x, y_{1}, \ldots, y_{n}\right) \in \mathbb{R}^{n+1}:|P(\zeta)| \leq Q^{-1}\right\} \\
& \chi_{B}(R):=\left\{\left(x, y_{1}, \ldots, y_{n}\right) \in \mathbb{R}^{n+1}:\left|P^{\prime}(\zeta)\right| \leq R\right\} \\
& \chi_{C}(Q):=\left\{\left(x, y_{1}, \ldots, y_{n}\right) \in \mathbb{R}^{n+1}:\left|y_{t}\right| \leq Q^{\frac{1}{n}}, \quad 1 \leq t \leq n\right\},
\end{aligned}
$$


where $P(\zeta)=x+\zeta y_{1}+\cdots+\zeta^{n} y_{n}$.

Then for sufficiently large $Q$ and all $R$ we have

$$
\operatorname{vol}\left(\chi_{\mathrm{A}}(\mathrm{Q}) \cap \chi_{\mathrm{B}}(\mathrm{R}) \cap \chi_{\mathrm{C}}(\mathrm{Q})\right) \leq \mathrm{ERQ}^{-\frac{1}{n}}
$$

with some constant $E=E(n, \zeta)$ independent of $Q$.

Proof. Note first that $\chi_{A}(Q)$ is bounded by the two translates of the fixed hyperplane $P(\zeta)=0$ by $Q^{-1}$ in direction orthogonal to $P(\zeta)=0$ to both sides of this hyperplane $P(\zeta)=0$. In particular $\chi_{A}(Q)$ converges to $P(\zeta)=0$ for $Q \rightarrow \infty$, which is not so important, however. Similarly, $\chi_{B}(R)$ is the space between two hyperplanes parallel to the hyperplane $P^{\prime}(\zeta)=0$ with distance $R$ in both directions from the hyperplane $P^{\prime}(\zeta)=0$. Note that with respect to any other fixed direction $v \in \mathbb{R}^{n+1}$ with $v \notin\{P: P(\zeta)=0\}$ resp. $v \notin\left\{P: P^{\prime}(\zeta)=0\right\}$ (which is equivalent to $P(v) \neq 0$ resp. $P^{\prime}(v) \neq 0$ ) $\chi_{A}(Q)$ resp. $\chi_{B}(R)$ has width at most $N_{1} Q^{-1}$ resp. $N_{2} R$ for fixed constants $N_{1}, N_{2}$ depending on $v$ but independent from $Q, R$. Throughout the proof we will make use of this for some given $v$ determined by $n, \zeta$.

Finally, $\chi_{C}(Q)$ just bounds the coordinates $\left(y_{1}, y_{2}, \ldots, y_{n}\right)$ of the vectors $\left(x, y_{1}, \ldots, y_{n}\right) \in$ $\mathbb{R}^{n+1}$ in dependence of $Q$.

Observe that by these restrictions for $\chi_{C}(Q)$ and in view of the left hand inequality in (2.6) we can assume $|x| \leq C(n, \zeta) Q^{\frac{1}{n}}$ for some constant $C(n, \zeta)$ independent of $Q$ without loss of generality. Thus every coordinate $\left(x, y_{1}, \ldots, y_{n}\right)$ of a point in $\chi_{A}(Q) \cap \chi_{B}(R) \cap \chi_{C}(Q)$ is bounded by $C(n, \zeta) Q^{\frac{1}{n}}$. Consequently for every $Q>1$ any rotation of the set $\chi_{A}(Q) \cap$ $\chi_{B}(R) \cap \chi_{C}(Q)$ lies in the centralsymmetric cube with side length $\sqrt{n} C(n, \zeta) Q^{\frac{1}{n}}$ and surfaces parallel to the hyperplanes $x=0, y_{1}=0, \ldots, y_{n}=0$ given by

$$
\kappa(Q):=\left\{\left(x, y_{1}, \ldots, y_{n}\right):|x| \leq \sqrt{n} C(n, \zeta) Q^{\frac{1}{n}},\left|y_{t}\right| \leq \sqrt{n} C(n, \zeta) Q^{\frac{1}{n}}, 1 \leq t \leq k\right\} .
$$

We apply a rotation $\varpi$ on $\mathbb{R}^{n+1}$ in such a way that the hyperplane $P(\zeta)=0$ is sent to the hyperplane $H_{0}$ defined by $x=0$. Let $H_{1}$ be the image of the hyperplane $P^{\prime}(\zeta)=0$ under $\varpi$. Clearly $H_{0} \neq H_{1}$ as $P^{\prime}(\zeta)$ has lower degree than $P(\zeta)$ and hence the intersection $H_{0} \cap H_{1}$ has dimension $(n-1)$. As rotations preserve volumes,

$$
\operatorname{vol}\left(\varpi\left(\chi_{\mathrm{A}}(\mathrm{Q}) \cap \chi_{\mathrm{B}}(\mathrm{R}) \cap \chi_{\mathrm{C}}(\mathrm{Q})\right)\right)=\operatorname{vol}\left(\chi_{\mathrm{A}}(\mathrm{Q}) \cap \chi_{\mathrm{B}}(\mathrm{R}) \cap \chi_{\mathrm{C}}(\mathrm{Q})\right) .
$$

Moreover note also that intersecting $\chi_{A}(Q) \cap \chi_{B}(R) \cap \chi_{C}(Q)$ with $\kappa(Q)$ doesn't change the volume as stated (we may replace $\chi_{C}(Q)$ by $\kappa(Q)$ ).

More generally, for real numbers $a, b$ define

$$
\begin{aligned}
H_{0, a} & :=\varpi(\{P: P(\zeta)=a\})=\left\{\left(x, y_{1}, \ldots, y_{n}\right) \in \mathbb{R}^{n+1}: x=a\right\}, \\
H_{1, b} & :=\varpi\left(\left\{P: P^{\prime}(\zeta)=b\right\}\right),
\end{aligned}
$$

such that in particular $H_{0}=H_{0,0}, H_{1}=H_{1,0}$.

From the preliminary descriptions of $\chi_{A}(Q), \chi_{B}(R)$ we easily see

$$
\begin{aligned}
& \varpi\left(\chi_{A}(Q)\right)=\bigcup_{a \in\left[-Q^{-1}, Q^{-1}\right]} H_{0, a}, \\
& \varpi\left(\chi_{B}(Q)\right)=\bigcup_{b \in[-R, R]} H_{1, b},
\end{aligned}
$$

and by construction of $\kappa(Q)$ we conclude

$$
\varpi\left(\chi_{A}(Q) \cap \chi_{B}(R) \cap \chi_{C}(Q)\right) \subset\left(\cup_{a \in\left[-Q^{-1}, Q^{-1}\right]} H_{0, a}\right) \bigcap\left(\cup_{b \in[-R, R]} H_{1, b}\right) \bigcap \kappa(Q) .
$$

In view of (2.16) and since rotations don't change the volume it is sufficient to prove the upper estimate $E R Q^{-\frac{1}{n}}$ for the volume of the right hand side of (2.16), i.e.

$$
\operatorname{vol}\left(\left(\cup_{\mathrm{a} \in\left[-\mathrm{Q}^{-1}, \mathrm{Q}^{-1}\right]} \mathrm{H}_{0, \mathrm{a}}\right) \bigcap\left(\cup_{\mathrm{b} \in[-\mathrm{R}, \mathrm{R}]} \mathrm{H}_{1, \mathrm{~b}}\right) \bigcap \kappa(\mathrm{Q})\right) \leq \mathrm{ERQ}^{-\frac{1}{\mathrm{n}}},
$$


to establish the assertions of the Lemma.

In order to do this we use Fubini's Theorem twice. We first give upper bounds for the $(n-1)$ dimensional volumes of the intersections $H_{0, a} \cap H_{1, b} \cap \kappa(Q)$, then apply Fubini' Theorem to derive upper bounds for the $n$-dimensional volumes of $H_{0, a} \cap \bigcup_{b \in[-R, R]} H_{1, b} \cap \kappa(Q)$ for every $a \in\left[-Q^{-1}, Q^{-1}\right]$ and then again apply Fubini's Theorem by integrating these $n$-dimensional volumes along the $x$-axis to finally derive the required upper bound.

Clearly, the $(n-1)$-dimensional volume of $H_{0} \cap H_{1} \cap \kappa(Q)$ is proportional to $Q^{\frac{n-1}{n}}$, let's say $\operatorname{vol}\left(\mathrm{H}_{0} \cap \mathrm{H}_{1} \cap \kappa(\mathrm{Q})\right)=\mathrm{DQ}^{\frac{\mathrm{n}-1}{\mathrm{n}}}$ for a constant $D$ depending only on the angle between $H_{0}$ and $H_{1}$ which is determined by $n, \zeta$ (in particular independent of $Q$ ). Similarly we see that we can find a constant $D_{0}=D_{0}(n, \zeta)$ such that simultaneously for all $a, b \in \mathbb{R}$ we have

$$
\operatorname{vol}\left(\mathrm{H}_{0, \mathrm{a}} \cap \mathrm{H}_{1, \mathrm{~b}} \cap \kappa(\mathrm{Q})\right) \leq \mathrm{D}_{0} \mathrm{Q}^{\frac{\mathrm{n}-1}{\mathrm{n}}}, \quad \mathrm{a}, \mathrm{b} \in \mathbb{R},
$$

as all $H_{0, a} \cap H_{1, b}$ are $(n-1)$-dimensional subspaces in a $n$-dimensional cube with side length proportional to $Q^{\frac{1}{n}}$.

Since $\chi_{B}(R)$ (and so $\varpi\left(\chi_{B}(R)\right)=\cup_{b \in[-R, R]} H_{1, b}$ too) has width $R$ in direction orthogonal to $H_{1, b}$, and in view of (2.18), we infer that

$$
\operatorname{vol}_{\mathrm{n}}\left(\mathrm{H}_{0, \mathrm{a}} \bigcap\left(\cup_{\mathrm{b} \in[-\mathrm{R}, \mathrm{R}]} \mathrm{H}_{1, \mathrm{~b}}\right) \bigcap \kappa(\mathrm{Q})\right) \leq \mathrm{D}_{0} \mathrm{Q}^{\frac{\mathrm{n}-1}{\mathrm{n}}} \cdot \mathrm{D}_{1} \mathrm{R}, \quad \mathrm{a} \in \mathbb{R}
$$

with some constant $D_{1}$ depending only on the angle between the $x$-axis and $H_{1}$, which itself is determined by $n, \zeta$. So we have estimated the $n$-dimensional volume of $\varpi\left(\chi_{B}(R) \cap \chi_{C}(Q)\right)=$ $\left(\cup_{b \in[-R, R]} H_{1, b}\right) \cap \kappa(Q)$ in every hyperplane $H_{0, a}$. Observe that $\varpi\left(\chi_{A}(Q) \cap \chi_{B}(R) \cap \chi_{C}(Q)\right)$ has width $m Q^{-1}$ in direction of the $x$-axis with a constant $m$ depending only on the angle between the hyperplane $P(\zeta)=0$ and the hyperplane $H_{0}$, which is again determined by $n, \zeta$ (so in particular independent of $Q$ ). We apply Fubini's Theorem to (2.19) and conclude

$\operatorname{vol}\left(\left(\cup_{\mathrm{a} \in\left[-\mathrm{Q}^{-1}, \mathrm{Q}^{-1}\right]} \mathrm{H}_{0, \mathrm{a}}\right) \bigcap\left(\cup_{\mathrm{b} \in[-\mathrm{R}, \mathrm{R}]} \mathrm{H}_{1, \mathrm{~b}}\right) \bigcap \kappa(\mathrm{Q})\right) \leq \mathrm{mQ} \mathrm{Q}^{-1} \cdot \mathrm{D}_{0} \mathrm{D}_{1} \mathrm{Q}^{\frac{\mathrm{n}-1}{\mathrm{n}}} \mathrm{R}=\mathrm{mD}_{0} \mathrm{D}_{1} \mathrm{Q}^{-\frac{1}{\mathrm{n}}} \mathrm{R}$.

Inequality (2.17) and hence the assertion of the Lemma follows with $E=E(n, \zeta):=m D_{0} D_{1}$.

Corollary 2.2. In the context of the first part of the proof of Theorem 1.1 (ie (2.2) holds) we have $c(Q) \geq B$ for some constant $B$ uniformly in $Q$, in particular (2.13) holds.

Proof. By definition of $c(Q)$ we have $c(Q)=R(Q) Q^{-\frac{1}{n}}$ for $R=R(Q)$ that satisfies

$$
\operatorname{vol}\left(\chi_{\mathrm{A}}(\mathrm{Q}) \cap \chi_{\mathrm{B}}(\mathrm{R}) \cap \chi_{\mathrm{C}}(\mathrm{Q})\right)=\frac{2^{\mathrm{n}+1}}{2(\mathrm{n}+1) !} .
$$

We may apply Lemma 2.1 and with respect to the constant $E=E(n, \zeta)$ of this lemma this yields

$$
\frac{2^{n+1}}{2(n+1) !} \leq E R(Q) Q^{-\frac{1}{n}}
$$

or equivalently $B \cdot Q^{\frac{1}{n}} \leq R(Q)=Q^{\frac{1}{n}} c(Q)$ with $B:=\frac{2^{n+1}}{2(n+1) ! E}$ for all $Q>1$ and we conclude $c(Q) \geq B$ uniformly in the parameter $Q$.

Thus we have established Theorem 1.1 and consequently Theorem 1.2 .

Remarks: 1) One can show $\operatorname{vol}\left(\chi_{\mathrm{A}}(\mathrm{Q}) \cap \chi_{\mathrm{B}}(\mathrm{R}) \cap \chi_{\mathrm{C}}(\mathrm{Q})\right) \geq \mathrm{FRQ}^{-\frac{1}{n}}$ for some constant $F=F(n, \zeta)$ independent from $Q$ with a proof similar to the one of Lemma 2.1. So by arguments very similar to those in the proof of Corollary 2.2 we have that in fact $c(Q)$ is also uniformly bounded above by a positive constant and consequently in combination with (2.13) we actually have $\lim _{Q \rightarrow \infty} \log _{Q} c(Q)=0$.

2) Observe that all the constants occuring throughout the proof of Lemma 2.1 can be estimated explicitely in dependence of $n, \zeta$ so we can write $c(Q) \leq K(n, \zeta)$ with an effective constant $K(n, \zeta)$ in (2.8), (2.11). However, the $\epsilon$-term in the exponent of (2.7), (2.10) doesn't allow any improvements in (2.14), (2.15) for any given $n, \zeta$. 
3) Lemma 15 Chap. 3 of $\S 3$ in $[8$ states that for a polynomial $P$ of degree $D$ and length $L$ and with zero $\alpha$ satisfying $|\zeta-\alpha| \leq 1$

holds, leading to the well known result

$$
|P(\zeta)| \leq|\zeta-\alpha| \cdot L D(1+|\zeta|)^{D-1}
$$

$$
w_{n}^{*}(\zeta) \leq w_{n}(\zeta)=w_{n, 1}(\zeta)
$$

So $w_{n}^{*}(\zeta)$ can be bounded above in terms of the approximation constants $w_{n, j}(\zeta)$ which reverses the direction of the estimates in Theorem 1.1

2.3. Estimates for $w_{n}^{*}(\zeta)$ depending on $n$ only. A famous result of Wirsing [10] states

$$
w_{n}^{*}(\zeta) \geq \frac{w_{n}(\zeta)+1}{2} .
$$

We combine the results of Theorem 1.1, 1.2 with (2.20) to give lower bounds for $w_{n}^{*}(\zeta)$ uniformly in $\zeta$ not algebraic of degree $\leq n$. In order to do this, we use the funcions $\psi_{n, j}^{*}$ which have the useful property

$$
\left|\sum_{j=1}^{n+1} \psi_{n, j}^{*}(Q)\right| \leq \frac{C(n)}{\log (Q)},
$$

which follows from (1.19) and (1.14), and $\psi_{n, j}^{*}$ relate to the constants $w_{n, j}(\zeta)$. To get a slightly better result we will also use the dual version of (1.7): indeed from (1.7) and (1.16) it follows that for $\zeta$ not algebraic of degree $\leq n$ we have

$$
\underline{\psi}_{n, j+1}^{*} \leq \bar{\psi}_{n, j}^{*}, \quad 1 \leq j \leq n .
$$

The following preliminary Proposition is a very easy consequence of (1.14) and (2.22).

Proposition 2.3. Let $n \geq 2$ be an integer. Then for $\zeta$ not algebraic of degree $\leq n$ the relation

$$
\underline{\psi}_{n, 1}^{*} \leq-\frac{2}{n-1} \underline{\psi}_{n, n+1}^{*}
$$

holds for the approximation constants $\psi_{n, 1}^{*}, \psi_{n, n+1}^{*}$ associated to $\left(\zeta, \zeta^{2}, \ldots, \zeta^{n}\right)$.

Proof. By definition for any $\epsilon>0$ and sufficiently large $Q$ we have $\psi_{n, n+1}^{*}(Q) \geq \underline{\psi}_{n, n+1}^{*}-\epsilon$. By (2.22) we also have $\underline{\psi}_{n, n+1}^{*} \leq \bar{\psi}_{n, n}^{*}$, so that there exist arbitrarily large values of $Q$ such that $\psi_{n, n}^{*}(Q) \geq \psi_{n, n+1}^{*}(Q)-\epsilon$. Combining these observations yields arbitrarily large values $Q$, such that $\psi_{n, n}^{*}(Q)+\psi_{n, n+1}^{*}(Q) \geq 2 \underline{\psi}_{n, n+1}^{*}(Q)-2 \epsilon$.

On the other hand, for any $Q$ we have $(n-1) \psi_{n, 1}^{*}(Q) \leq \sum_{j=1}^{n-1} \psi_{n, j}^{*}(Q)$, in particular for those values $Q$ with the property $\psi_{n, n}^{*}(Q)+\psi_{n, n+1}^{*}(Q) \geq 2 \underline{\psi}_{n, n+1}^{*}(Q)-2 \epsilon$. The assertion of the Proposition follows with (2.21) und $\epsilon \rightarrow 0$.

An application of Proposition 2.3 together with Wirsing's result yields

Corollary 2.4. Let $n \geq 2$ be an integer. Then for $\zeta$ not algebraic of degree $\leq n$ the approximation constant $\bar{w}_{n}^{*}(\zeta)$ of $\left(\zeta, \zeta^{2}, \ldots, \zeta^{n}\right)$ is bounded below as follows

$$
w_{n}^{*}(\zeta) \geq \frac{1}{4}\left(n+1+\sqrt{n^{2}+10 n-7}\right)=: \mathscr{U}(n) .
$$

For $n \rightarrow \infty$ we have the asymptotical behaviour $\mathscr{U}(n)=\frac{n}{2}+\frac{3}{2}+o(1)$.

Proof. From (1.17) we have

$$
\underline{\psi}_{n, n+1}^{*}=\frac{n-w_{n, n+1}(\zeta)}{n\left(w_{n, n+1}(\zeta)+1\right)}
$$


Dividing the right hand side of (2.24) by $(n-1)$ and combining it with the estimate from Proposition 2.3 gives an upper bound for $\underline{\psi}_{n, 1}^{*}$ in terms of $w_{n, n+1}(\zeta)$. Using this expression in (1.17) and applying Wirsing's result (2.20) leads to

$$
w_{n}^{*}(\zeta) \geq \frac{w_{n}(\zeta)+1}{2} \geq \frac{n+1}{2} \cdot \frac{1}{1-\frac{2}{n-1} \frac{n-w_{n, n+1}(\zeta)}{w_{n, n+1}(\zeta)+1}} .
$$

On the other hand we have the lower bound $w_{n, n+1}(\zeta)$ for $w_{n}^{*}(\zeta)$ by Theorem 1.1, so

$$
w_{n}^{*}(\zeta) \geq \max \left\{\frac{n+1}{2} \cdot \frac{1}{1-\frac{2}{n-1} \frac{n-w_{n, n+1}(\zeta)}{w_{n, n+1}(\zeta)+1}}, w_{n, n+1}(\zeta)\right\} .
$$

It's not hard to see that the left hand term in the maximum decreases as $w_{n, n+1}(\zeta)$ increases, so the minimum is attained at the value $w_{n, n+1}(\zeta)>0$ where both expressions in the maximum coincide. This leads to a quadratic equation and after basic simplifications finally yields $\mathscr{U}(n)$ as the minimum lower bound for $w_{n}^{*}(\zeta)$. Checking the asymptotics for $\mathscr{U}(n)$ is a standard calculation.

Remarks: 1) Lemma 1 on page 46 in [5] states that in the present case of simultaneous approximation of $\left(\zeta, \zeta^{2}, \ldots, \zeta^{n}\right)$ of $\zeta$ not algebraic of degree $\leq\left\lceil\frac{n}{2}\right\rceil$ the approximation constant $\widehat{w}_{n}^{\prime}(\zeta)$ is bounded above by $\left\lceil\frac{n}{2}\right\rceil^{-1}$. Applying this to (1.22) and Theorem 1.1 we immediately derive

$$
w_{n}^{*}(\zeta) \geq\left\lceil\frac{n}{2}\right\rceil
$$

and slight refinements in combination with Wirsing's result can be derived similarly as in Corollary 2.4. However, the results of Corollary 2.4 are a little stronger.

2 ) Note that there exists no nontrivial upper bound for the value $w_{n}^{\prime}(\zeta)$ (as for $\widehat{w}_{n}^{\prime}(\zeta$ ) in Remark 1) even in the present special case of simultaneous approximation of $\left(\zeta, \zeta^{2}, \ldots, \zeta^{n}\right)$. Indeed, we can have $w_{n}^{\prime}(\zeta)=\infty$ (which is equivalent to $\widehat{w}_{n, n+1}(\zeta)=0$ by (1.22) $)$, taking $\zeta=\sum_{l \geq 1} 10^{-l !}$ for example. So we cannot use Theorems 1.11 .2 to give nontrivial bounds for the approximation constant $\widehat{w}_{n}^{*}(\zeta)$ as easily as above.

Moreover, no analogue of (2.20) for $\widehat{w}_{n}^{*}(\zeta)$ seems to be known. Bugeaud and Laurent established in Theorem 2.1 in [1] for $\zeta$ not algebraic of degree $\leq n$ the inequality

$$
\widehat{w}_{n}^{*}(\zeta) \geq \frac{w_{n}(\zeta)}{w_{n}(\zeta)-n+1}
$$

which we can combine with Theorem 1.1 to get

$$
\widehat{w}_{n}^{*}(\zeta) \geq \max \left\{\frac{w_{n}(\zeta)}{w_{n}(\zeta)-n+1}, \widehat{w}_{n, n+1}(\zeta)\right\} .
$$

However, any number $\zeta$ with $w_{n}^{\prime}(\zeta)=\infty$ (for instance again $\zeta=\sum_{l \geq 1} 10^{-l !}$ ) automatically yields $w_{n}(\zeta)=\infty$ (which follows easily from the definition of $w_{n}(\zeta), w_{n}^{\prime}(\zeta)$ or alternatively from Khinchins transference principle $w_{n}(\zeta) \geq(n-1) w_{n}^{\prime}(\zeta)+n-2$, see [4]). For such $\zeta$, by (1.22) we also have $\widehat{w}_{n, n+1}(\zeta)=0$ though, so in this case (2.25) only leads to the very weak bound $\widehat{w}_{n}^{*}(\zeta) \geq 1$. 
TWO ESTIMATES CONCERNING CLASSICAL DIOPHANTINE APPROXIMATION CONSTANTS

\section{REFERENCES}

[1] Y. Bugeaud, M. Laurent: Exponents of Diophantine approximation and Sturmian continued fractions, Ann. Inst. Fourier (Grenoble) 55 (2005), no. 3, p. 773-804

[2] H. Davenport, W.M. Schmidt: Approximation to real numbers by algebraic integers, Acta Arith. 15 (1969), p. 393-416

[3] P.M. Gruber, C.G. Lekkerkerker: Geometry of numbers, North-Holland Verlag (1987)

[4] Y.A. Khintchine [A. Ya. Khinchin]: Zur metrischen Theorie der diophantischen Approximationen, Math. Z. 24 (1926), 706-714

[5] M. Laurent: On simultaneous rational approximation to successive powers of a real number, Indag. Math. (N.S.) 14 (2003), no. 1,p. 45-53

[6] W.M. Schmidt, L. Summerer: Parametric geometry of numbers and applications, Acta Arithm. 140.1 (2009)

[7] W.M. Schmidt, L. Summerer: Diophantine approximation and parametric geometry of numbers, Monatshefte für Mathematik Vol 169, Issue 1 (2013) p. 54-107

[8] T. Schneider: Einführung in die transzendenten Zahlen, Springer-Verlag Berlin (1957)

[9] M. Waldschmidt: Report on some recent advances in Diophantine approximation (2009)

[10] E. Wirsing: Approximation mit algebraischen Zahlen beschränkten Grades, J. Reine Angew. Math. 206 1960 67-77. \# 79 